Article

\title{
Metformin Restores Parkin-Mediated Mitophagy, Suppressed by Cytosolic p53
}

\author{
Young Mi Song ${ }^{1,+}$, Woo Kyung Lee ${ }^{2,+}$, Yong-ho Lee ${ }^{2}$, Eun Seok Kang ${ }^{2}$, Bong-Soo Cha ${ }^{2}$ and \\ Byung-Wan Lee ${ }^{2, *}$
}

Received: 9 November 2015; Accepted: 13 January 2016; Published: 16 January 2016

Academic Editor: Masato Matsuoka

1 Brain Korea 21 PLUS Project for Medical Science, Yonsei University College of Medicine, 03722 Seoul, Korea; ymsong1225@gmail.com

2 Division of Endocrinology and Metabolism, Department of Internal Medicine, Yonsei University College of Medicine, 03722 Seoul, Korea; lewoky7@yuhs.ac (W.K.L.); yholee@yuhs.ac (Y.L.); edgo@yuhs.ac (E.S.K.); bscha@yuhs.ac (B.S.C.)

* Correspondence: bwanlee@yuhs.ac; Tel.: +82-2-2228-1938; Fax: +82-2-393-6884

+ These authors contributed equally to this work.

\begin{abstract}
Metformin is known to alleviate hepatosteatosis by inducing $5^{\prime}$ adenosine monophosphate (AMP)-kinase-independent, sirtuin 1 (SIRT1)-mediated autophagy. Dysfunctional mitophagy in response to glucolipotoxicities might play an important role in hepatosteatosis. Here, we investigated the mechanism by which metformin induces mitophagy through restoration of the suppressed Parkin-mediated mitophagy. To this end, our $o b / o b$ mice were divided into three groups: (1) ad libitum feeding of a standard chow diet; (2) intraperitoneal injections of metformin $300 \mathrm{mg} / \mathrm{kg}$; and (3) $3 \mathrm{~g} /$ day caloric restriction (CR). HepG2 cells were treated with palmitate (PA) plus high glucose in the absence or presence of metformin. We detected enhanced mitophagy in $o b / o b$ mice treated with metformin or $\mathrm{CR}$, whereas mitochondrial spheroids were observed in mice fed ad libitum. Metabolically stressed $o b / o b$ mice and PA-treated HepG2 cells showed an increase in expression of endoplasmic reticulum (ER) stress markers and cytosolic p53. Cytosolic p53 inhibited mitophagy by disturbing the mitochondrial translocation of Parkin, as demonstrated by immunoprecipitation. However, metformin decreased ER stress and p53 expression, resulting in induction of Parkin-mediated mitophagy. Furthermore, pifithrin- $\alpha$, a specific inhibitor of p53, increased mitochondrial incorporation into autophagosomes. Taken together, these results indicate that metformin treatment facilitates Parkin-mediated mitophagy rather than mitochondrial spheroid formation by decreasing the inhibitory interaction with cytosolic p53 and increasing degradation of mitofusins.
\end{abstract}

Keywords: mitophagy; p53; Parkin; mitochondrial spheroid; metformin

\section{Introduction}

A thorough understanding of the molecular machineries involved in the underlying pathophysiology and pharmacodynamics are crucial to the treatment of metabolic disorders including fatty liver disease. We recently elucidated a novel lipophagic mechanism by which metformin, a kind of anti-diabetic drug that disturbs mitochondrial complex I of the electron transport chain, alleviates non-alcoholic fatty liver disease (NAFLD) by inducing the sirtuin class of histone/protein deacetylases sirtuin 1 (SIRT1)-mediated autophagy independent of $5^{\prime}$ adenosine monophosphate-activated protein kinase (AMP-activated protein kinase) [1]. Of the intracellular organelles involved in this underlying pathophysiologic mechanism, the degradation of dysfunctional mitochondria and the reciprocal biogenesis of mitochondria, i.e., mitophagy, are areas of debate and investigation [2,3]. Although 
controversies remain as to the distinct machinery utilized for mitophagy in response to cell stressors, dysfunctional mitophagy in response to glucose and lipid toxicities in hepatocytes might play an important role in the accumulation of intracellular or extracellular oxidative stress, which causes NAFLD, ultimately leading to hepatocellular carcinoma [3-5]. In addition, recent studies have reported that cytosolic p53 interferes with mitochondrial integrity and mitophagy [6,7] by inhibiting the removal of damaged mitochondria through an inhibitory interaction with Parkin, thereby inducing mitochondrial dysfunction [8,9]. Based on these previous reports, we hypothesized and aimed to elucidate the mechanism by which metformin induces mitophagy through restoration of the Parkin-mediated mitophagy suppressed by increased cytosolic ER stress and p53.

\section{Results}

\subsection{Metformin Treatment Upregulates Mitophagy}

The electron microscopic findings resulting from ad libitum feeding of chow diets (Figure 1A-a), caloric restriction (CR) (Figure 1A-b), and ad libitum feeding of chow diets and $300 \mathrm{mg} / \mathrm{kg}$ metformin treatment (Figure 1A-c) in mice are shown. Electron microscopy readily detected spheroid mitochondria forming a clearly defined internal space surrounded by mitochondrial membranes in mice fed a standard chow diet (Figure 1A-a). The compressed mitochondria (white arrow), the lost cristae, and some portion of the matrix might connect the extra-mitochondrial space through a small orifice (black arrow) or form a lumen containing subcellular contents (asterisk). The white arrowheads indicate either autophagic double membranes surrounding abnormal mitochondria or an autophagic vacuole containing mitochondria (Figure 1A-c).

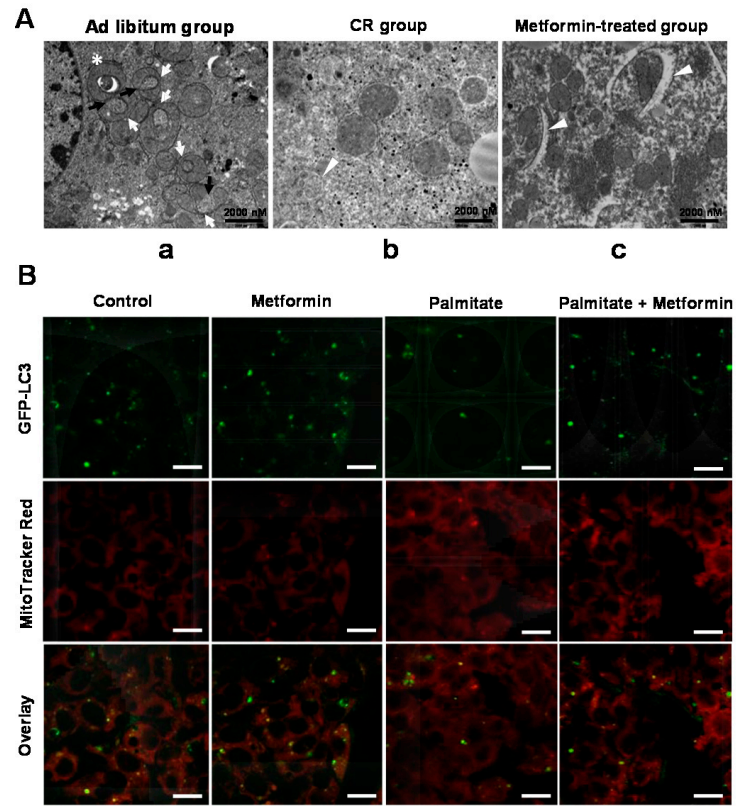

Figure 1. Metformin induced mitophagy. Electron microscopy was performed on hepatocytes from $o b / o b$ mice under ad libitum feeding of chow diet (A-a), caloric restriction (A-b), and ad libitum feeding of chow diet with $300 \mathrm{mg} / \mathrm{kg}$ metformin treatment (A-c). In the ad libitum group, spheroid mitochondria were readily detected. However, in the metformin-treated group, autophagic double membranes were detected, indicating mitophagy; (B) co-localization between a marker of autophagosomes (LC3) and a mitochondrial marker (MitoTracker Red) was assessed in HepG2 cells stably transfected with green fluorescent protein (GFP)-LC3. Although there was little co-localization between mitochondria and autophagosomes in the $0.25 \mathrm{mM}$ palmitate-treated HepG2 cells, LC3-labeled structures were seen surrounding the fragmented mitochondria in cells treated with $0.5 \mathrm{mM}$ metformin and $0.25 \mathrm{mM}$ palmitate. Scale bar $=20 \mu \mathrm{m}$. 
We assessed co-localization between a marker of autophagosomes, LC3, and a mitochondrial marker (MitoTracker Red) in HepG2 cells stably transfected with GFP-LC3. HepG2 cells ectopically expressing GFP-LC3 exhibited an increase in the number of punctate GFP-LC3 structures upon exposure to $0.5 \mathrm{mM}$ metformin. Furthermore, LC3-labeled structures were found surrounding the fragmented mitochondria in cells treated with metformin and palmitate, although there was little co-localization between mitochondria and autophagosomes in cells treated with $0.25 \mathrm{mM}$ palmitate alone (Figure 1B).

\subsection{Palmitate Increases but Metformin Decreases the Expression of p53 Protein}

To determine whether metformin down-regulated p53 expression, Western blotting was performed using the cytosolic fraction. Western blot analysis showed a dose-dependent increase in cytosolic p53 protein in response to palmitate in the presence of $35 \mathrm{mM}$ glucose in HepG2 cells (Figure 2A-a). In contrast, when metformin was added to the same conditions, p53 expression decreased in a dose-dependent manner (Figure 2A-b). Treatment of HepG2 cells with both $0.25 \mathrm{mM}$ palmitate and $35 \mathrm{mM}$ glucose significantly activated cytosolic p53 (1.0 \pm 0 vs. $1.226 \pm 0.028, p<0.0001)$. However, pretreatment with $0.5 \mathrm{mM}$ metformin significantly attenuated this gluco-lipid induced expression of p53 in HepG2 cells (1.226 \pm 0.028 vs. $1.078 \pm 0.038, p<0.05$, Figure 2B-a). To confirm the effects of metformin in an animal model, we performed Western blotting using hepatocytes from $o b / o b$ mice. As shown in Figure 2B-b, expression of p53 was significantly attenuated in both the CR- $(0.639 \pm 0.072, p<0.001)$ and metformin-treated groups $(0.783 \pm 0.114, p<0.05)$ compared to the ad libitum-fed group.

\subsection{Metformin Induces Parkin-Mediated Mitophagy through Inhibition of Glucolipotoxicity-Induced Cytosolic p53}

To highlight the impact of metformin on mitophagy, we assessed co-localization between Parkin and a mitochondrial marker (MitoTracker Red) using immunofluorescence staining, and performed cytosolic and mitochondrial fractionation experiments in HepG2 cells. In the immunofluorescence study, there was little co-localization between Parkin and mitochondria in cells treated with $0.25 \mathrm{mM}$ palmitate and $35 \mathrm{mM}$ glucose, whereas metformin restored the co-localization (yellow dots) between Parkin and mitochondria. In the fractionation experiments, Parkin translocation from the cytosol to mitochondria was attenuated in cells treated with $0.25 \mathrm{mM}$ palmitate and $35 \mathrm{mM}$ glucose, whereas metformin restored the Parkin translocation suppressed by glucolipotoxicity (Figure 2C). We also found a negative relationship between Parkin and mitofusin translocation in response to metformin treatment. To examine p53 and Parkin protein-protein interactions, the endogenous Parkin-p53 complex was observed in immunoprecipitates (IP) of Parkin and p53 (Figure 2D). Treatment of HepG2 cells with both $0.25 \mathrm{mM}$ palmitate and $35 \mathrm{mM}$ glucose significantly upregulated the expression of Parkin. However, pretreatment of HepG2 cells with $0.5 \mathrm{mM}$ metformin significantly attenuated the gluco-lipid induced expression of Parkin (Figure 2D-a); similar findings were also found using ad libitum-fed $o b / o b$ mice and $o b / o b$ mice treated with CR or metformin (Figure 2D-b). These results indicate the involvement of p53 in the significant decrease in Parkin-dependent mitophagy observed under diabetic conditions.

\subsection{Metformin Induces Parkin-Mediated Mitophagy through Inhibition of Glucolipotoxicity-Induced Endoplasmic Reticulum (ER) Stress}

To elucidate possible ER stress-mediated glucolipotoxicity in a hepatocyte cell line, we investigated the effects of palmitate with glucose and metformin on ER stress markers using Western blotting. We demonstrated that HepG2 cells treated with both $0.25 \mathrm{mM}$ palmitate and $35 \mathrm{mM}$ glucose for $24 \mathrm{~h}$ showed significant activation of the expression of ATF6 and Xbp-1. However, pretreatment with $0.5 \mathrm{mM}$ metformin attenuated the expression of ER stress markers in HepG2 cells (Figure 3A-a). Similar findings were noted in both the CR- and metformin-treated groups compared to the ad libitum-fed 
group (Figure 3A-b). In addition, we investigated this pathway using the traditional ER stressor thapsigargin. As shown in Figure 3B, HepG2 cells exposed to different concentrations (0, 0.1, 0.2, and $0.5 \mu \mathrm{M}$ ) of thapsigargin showed upregulated expression of ER stress markers and of p53 protein. Based on this finding, we utilized $0.1 \mu \mathrm{M}$ thapsigargin in subsequent experiments. Compared to the untreated controls, HepG2 cells exposed to $0.1 \mu \mathrm{M}$ thapsigargin for $24 \mathrm{~h}$ showed significantly decreased levels of viability $(100.0 \pm 2.95$ vs. $94.0 \pm 1.24, p<0.05)$ although there is no significant difference in viability in HepG2 cells exposed to $0.5 \mathrm{mM}$ metformin for $24 \mathrm{~h}$, as assessed by CCK-8. Pretreatment with $0.5 \mathrm{mM}$ metformin for $3 \mathrm{~h}$ significantly restored the HepG2 cell viability reduced by $0.1 \mu \mathrm{M}$ thapsigargin $(94.0 \pm 1.24$ vs. $101.1 \pm 2.18, p<0.001$, Figure $3 \mathrm{C}-\mathrm{a})$. To elucidate possible ER stress-mediated mitophagic dysfunction, we performed cytosolic and mitochondrial fractionation experiments in HepG2 cells. Parkin translocation from the cytosol to mitochondria was attenuated in cells treated with $0.1 \mu \mathrm{M}$ thapsigargin, whereas metformin restored the Parkin translocation suppressed by thapsigargin (Figure 3C-b,c).

\section{A}

a
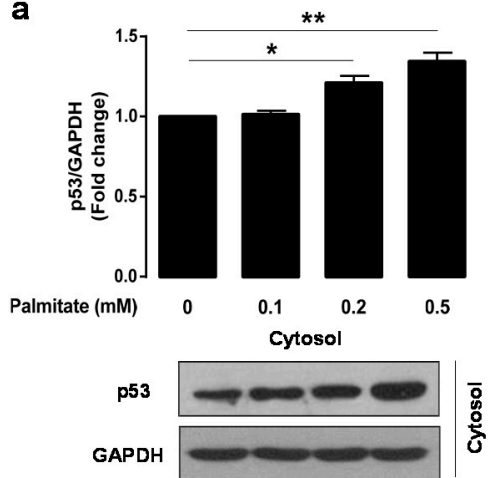

B

a

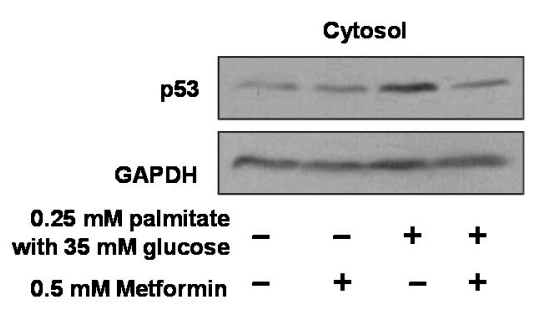

b

(a)

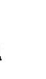

b
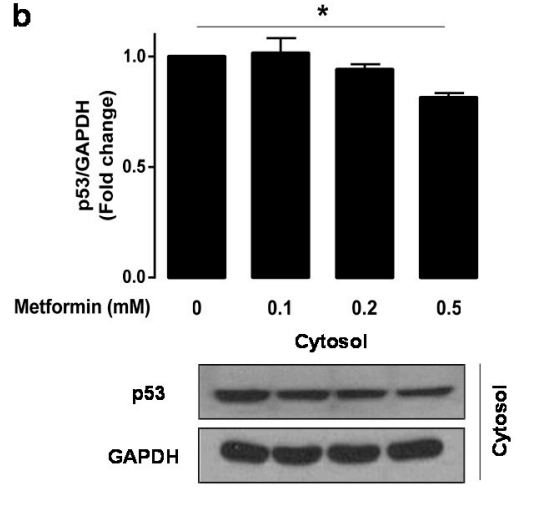
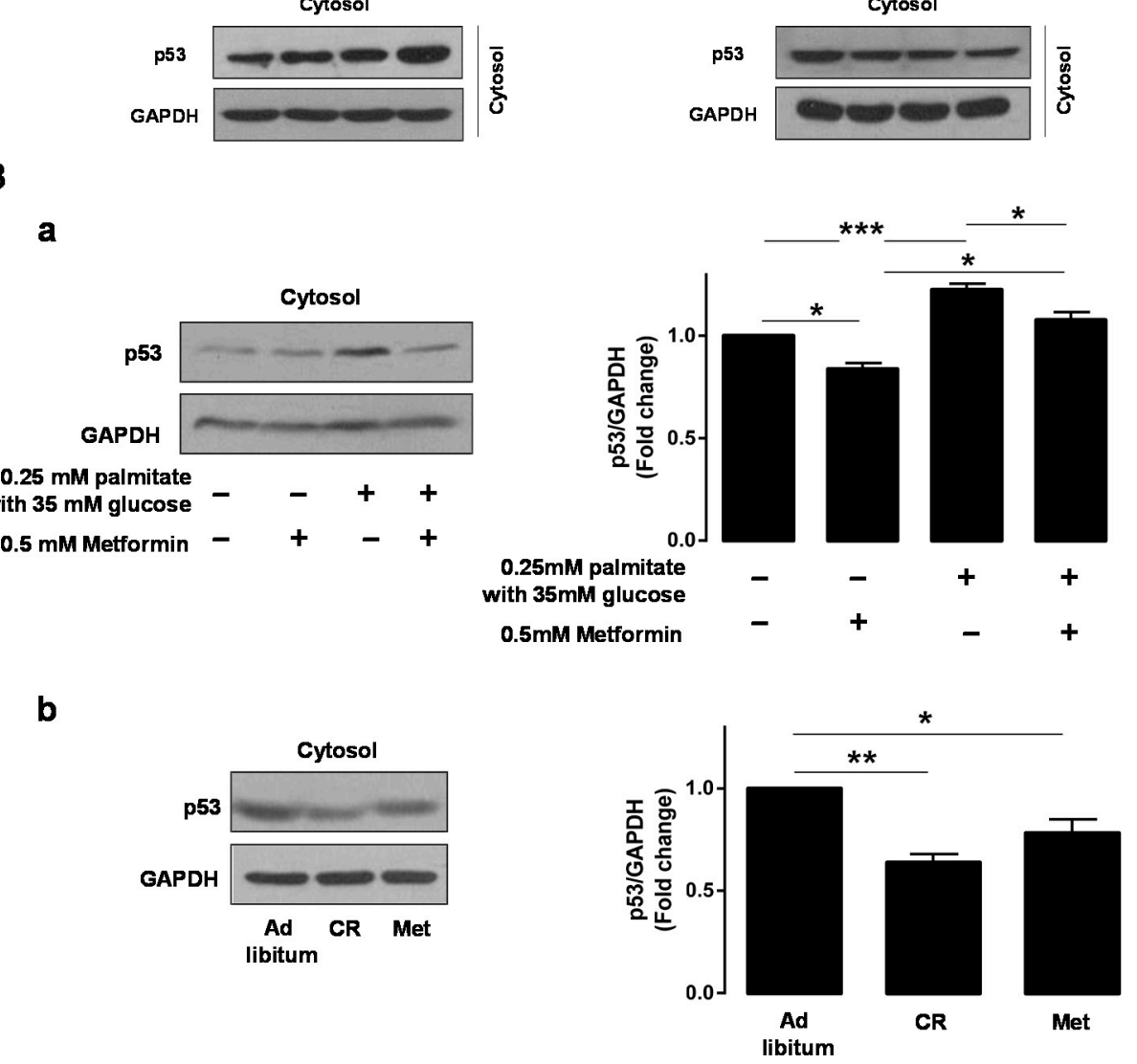

Figure 2. Cont. 
C
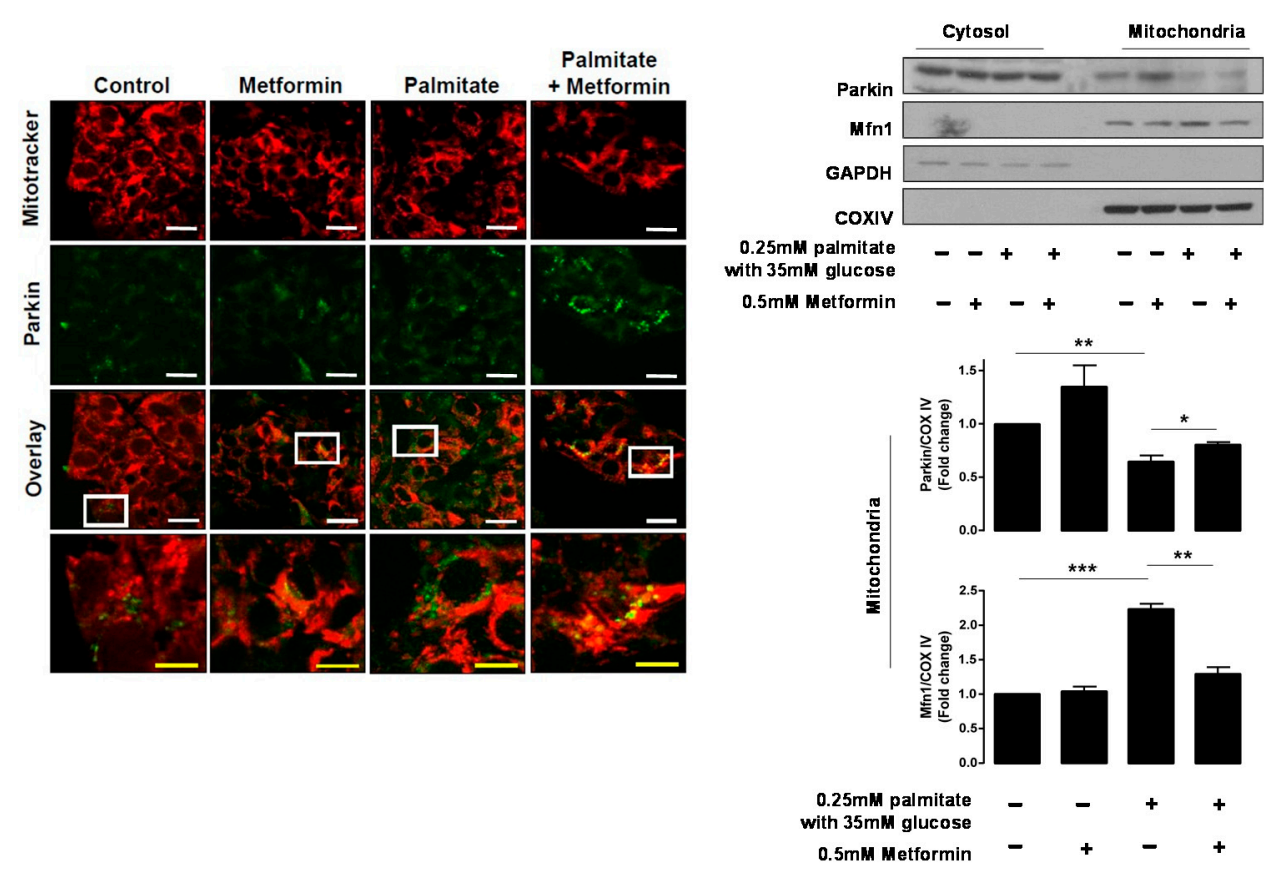

D

a
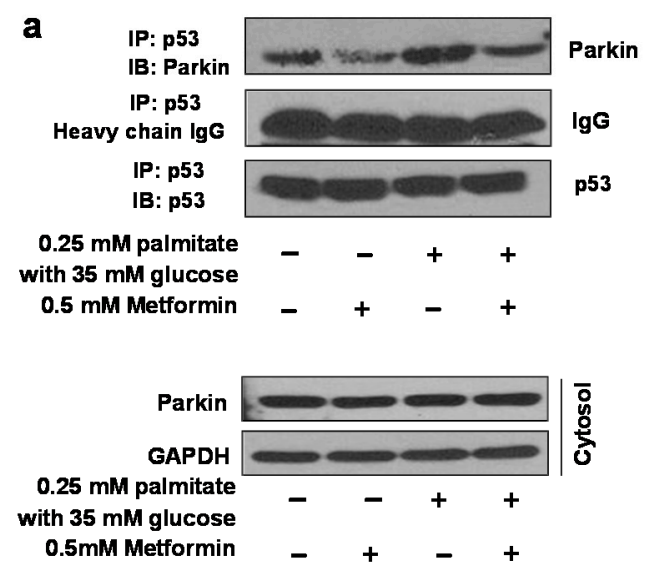

b

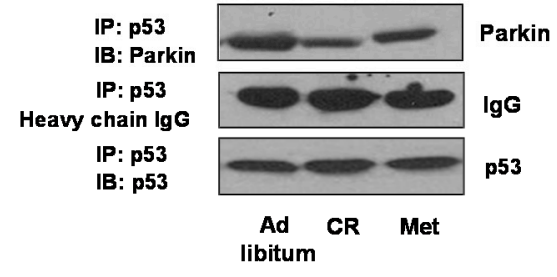

Parkin

GAPDH

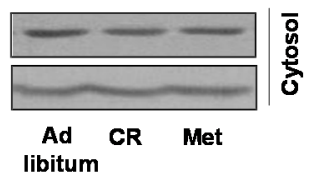

Figure 2. Metformin restored the Parkin-mediated mitophagy inhibited by glucolipotoxicity-induced cytosolic p53. Western blotting analysis was performed to assess cytosolic p53 levels with the cytosolic fractions isolated from palmitate- (A-a) or metformin- (A-b) and $35 \mathrm{mM}$ glucose-treated HepG2 cells; the expression of cytosolic p53 protein was assessed following $0.5 \mathrm{mM}$ metformin and/or $0.25 \mathrm{mM}$ palmitate with $35 \mathrm{mM}$ glucose treatment of HepG2 cells (B-a) or in hepatocytes from $o b / o b$ mice with ad libitum feeding of chow diet, caloric restriction, and ad libitum feeding of chow diet with $300 \mathrm{mg} / \mathrm{kg}$ metformin treatment (B-b); (C) Expression of Parkin (green) on mitochondria (MitoTracker Red) was observed with a confocal microscopy. The bottom panels show enlarged views of the boxed areas. The yellow dots indicate the mitochondria that co-localize with Parkin. The cytosolic and mitochondrial fractionation experiments demonstrated Parkin translocation from the cytosol to mitochondria depending on the conditions used in the HepG2 cells; a co-immunoprecipitation assay was conducted to investigate the interaction between p53 and Parkin, depending on the conditions used in the HepG2 cells (D-a) or in hepatocytes from mice (D-b). GAPDH or COX IV was used for normalization. Values displayed are mean $\pm \operatorname{SEM}(n=3$ independent experiments, respectively). Scale bar: white $=20 \mu \mathrm{m}$, yellow $=10 \mu \mathrm{m}$. Asterisks $\left({ }^{*} p<0.05,{ }^{* *} p<0.01,{ }^{* * *} p<0.001\right)$ indicate significant differences. Abbreviation: Mfn1, mitofusin1. 
A

a

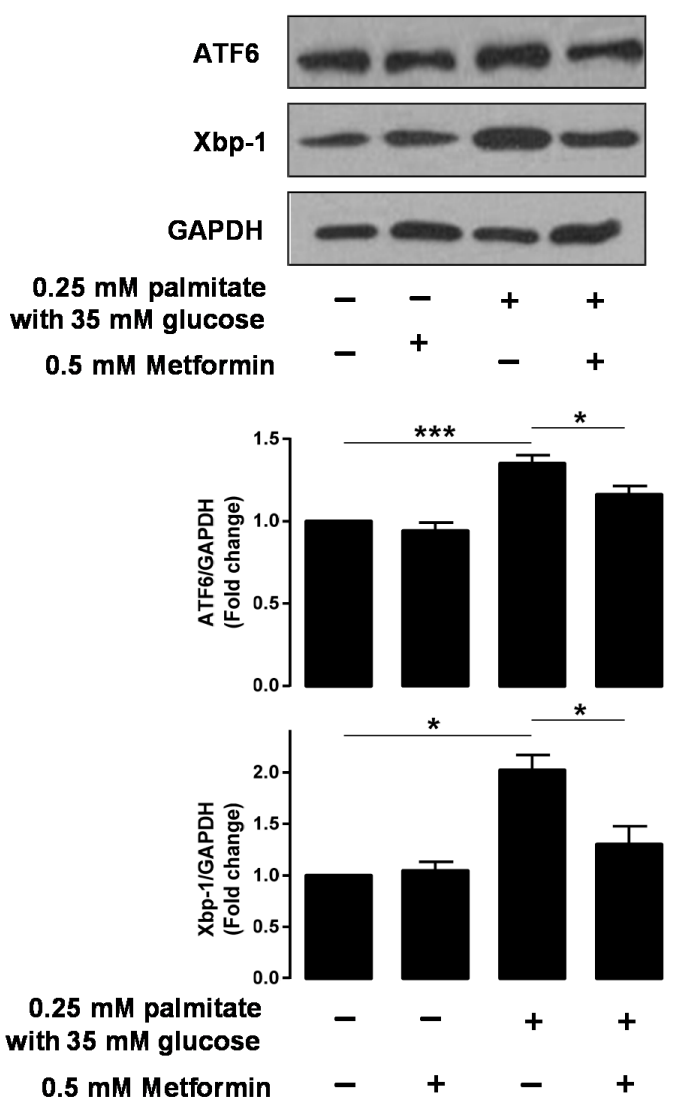

b
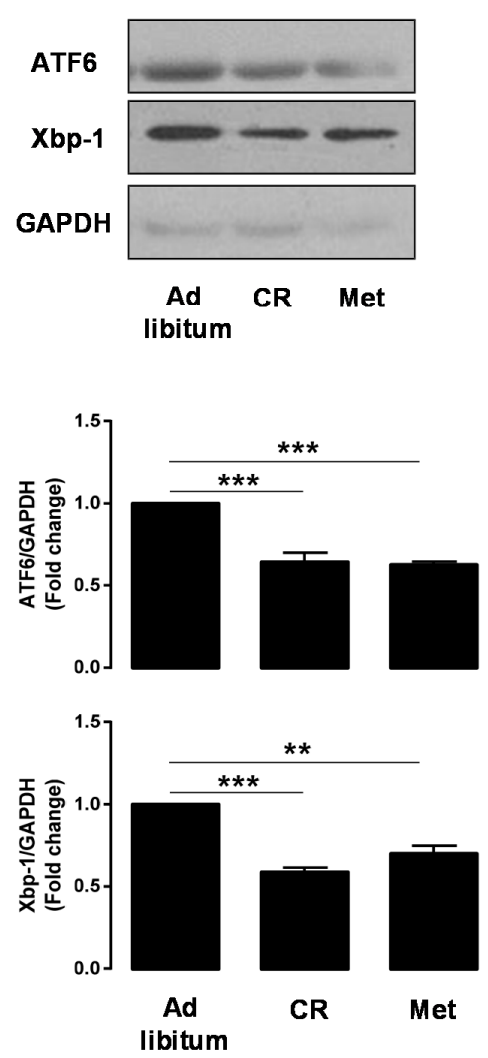

B
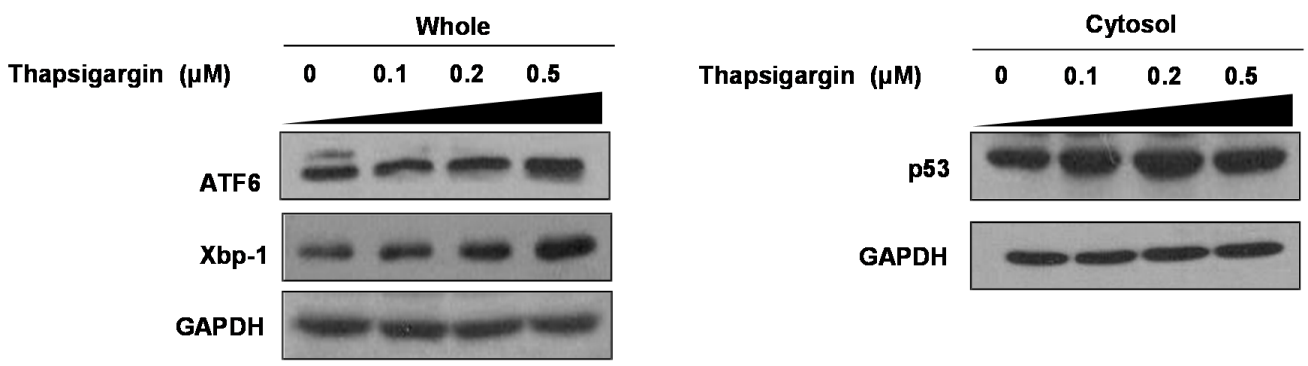

Figure 3. Cont. 
C

a

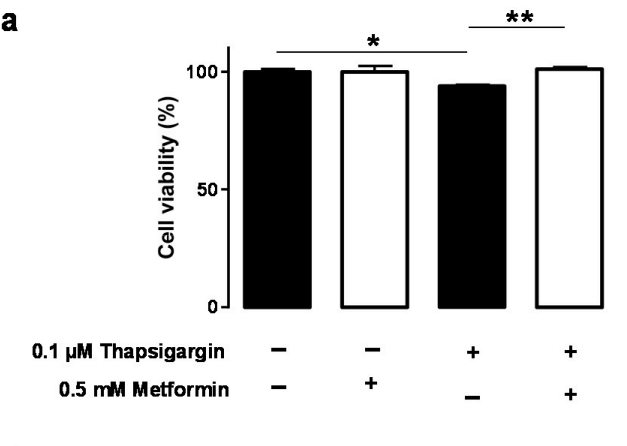

b

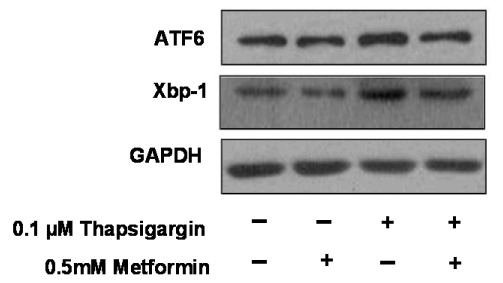

c

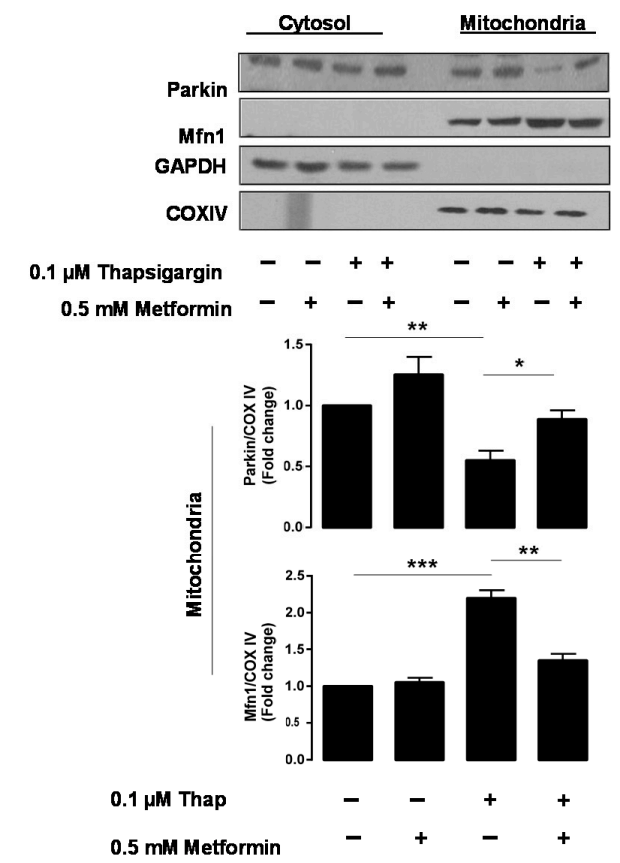

Figure 3. Metformin restored Parkin-mediated mitophagy inhibited by glucolipotoxicity-induced ER stress. The expression of ER stress markers was assessed following $0.5 \mathrm{mM}$ metformin and/or $0.25 \mathrm{mM}$ palmitate with $35 \mathrm{mM}$ glucose treatment of HepG2 cells (A-a) or in hepatocytes from $o b / o b$ mice with ad libitum feeding of chow diet, caloric restriction, or ad libitum feeding of chow diet with $300 \mathrm{mg} / \mathrm{kg}$ metformin treatment (A-b) using Western blotting analysis. Values displayed are mean $\pm \operatorname{SEM}(n=3$ independent experiments, respectively); (B) the expression of ER stress markers and p53 protein was assessed in response to thapsigargin in HepG2 cells; (C-a) cell viability was assessed in HepG2 cells exposed to $0.1 \mu \mathrm{M}$ thapsigargin and/or $0.5 \mathrm{mM}$ metformin using CCK-8. Values displayed are mean \pm SEM ( $n=5$ independent experiments); (C-b) the expression of an ER stress marker was also examined under these conditions; $(\mathbf{C}-\mathbf{c})$ the cytosolic and mitochondrial fractionation experiments demonstrated Parkin translocation from the cytosol to mitochondria, depending on the conditions used. Values displayed are mean \pm SEM ( $n=3$ independent experiments, respectively). GAPDH or COX IV was used for normalization. Asterisks $\left({ }^{*} p<0.05,{ }^{* *} p<0.01,{ }^{* * *} p<0.001\right)$ indicate significant differences. Abbreviation: Mfn1, mitofusin1; CCK-8, cell counting kit-8.

\subsection{A p53 Inhibitor Restores Parkin-Mediated Mitophagy Suppressed by Glucolipotoxicity}

To determine whether p53 actively mediates mitophagy dysfunction, we treated cells with pifithrin- $\alpha$ (PFT- $\alpha)$, a p53 inhibitor, to act as an ER stress reliever. Parkin translocation from the cytosol to mitochondria was attenuated in cells treated with $0.25 \mathrm{mM}$ palmitate and $35 \mathrm{mM}$ glucose, whereas PFT- $\alpha$ restored the Parkin translocation suppressed by glucolipotoxicity (Figure 4A). We additionally assessed co-localization between a marker of autophagosomes, LC3, and mitochondria in HepG2 cells stably transfected with GFP-LC3. LC3-labeled structures were observed surrounding the fragmented mitochondria in cells treated with PFT- $\alpha$ and palmitate, although there was little co-localization between mitochondria and autophagosomes in cells treated with $0.25 \mathrm{mM}$ palmitate alone. (Figure 4B). 
A a

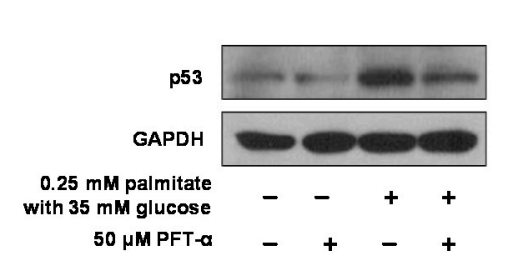

b

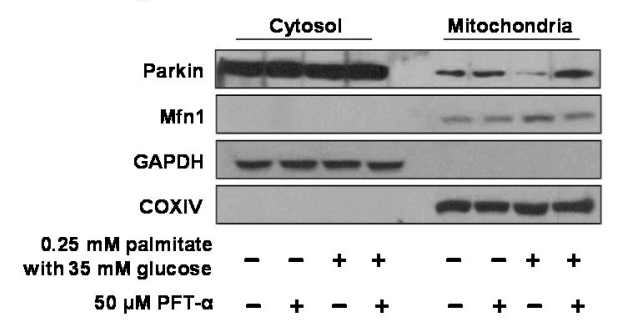

B

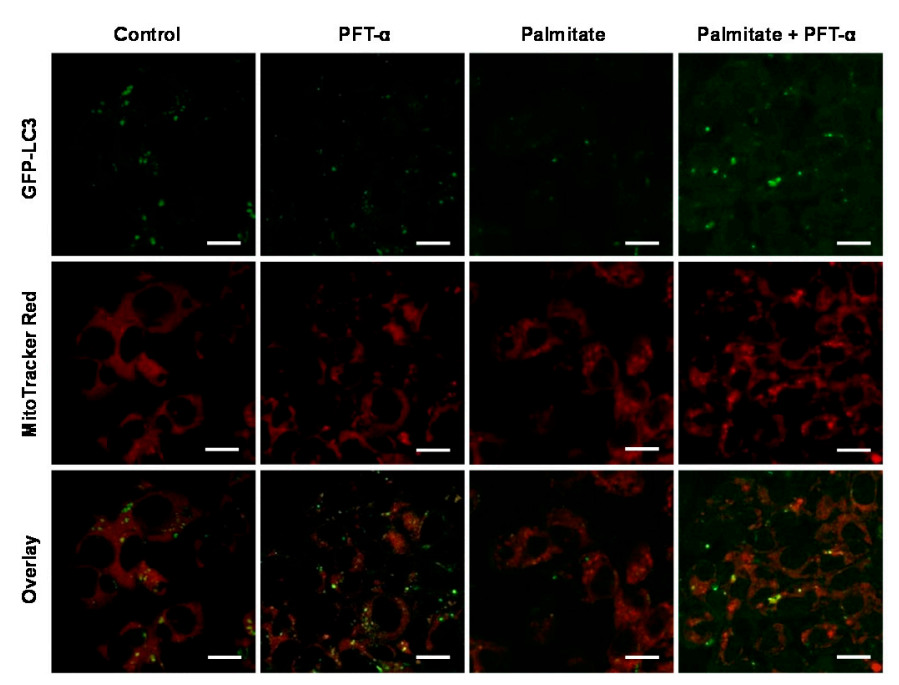

Figure 4. A p53 inhibitor restored the Parkin-mediated mitophagy inhibited by glucolipotoxicity. (A-a) the expression of cytosolic p53 was assessed in HepG2 cells treated with $50 \mu \mathrm{M}$ PFT- $\alpha$, a p53 inhibitor, and/or $0.25 \mathrm{mM}$ palmitate with $35 \mathrm{mM}$ glucose using Western blotting analysis; (A-b) the cytosolic and mitochondrial fractionation experiments demonstrated Parkin translocation from the cytosol to mitochondria, depending on the conditions used. Exposure time of Western blotting was long, compared to Figure 2C; (B) co-localization was assessed between a marker of autophagosomes (LC3) and a mitochondrial marker (MitoTracker Red) in HepG2 cells stably transfected with GFP-LC3, depending on the conditions used. LC3-labeled structures were observed surrounding the fragmented mitochondria in cells treated with PFT- $\alpha$ and palmitate, although there was little co-localization between mitochondria and autophagosomes in cells treated with $0.25 \mathrm{mM}$ palmitate alone. Scale bar $=20 \mu \mathrm{m}$. Abbreviation: Mfn1, mitofusin1; PFT- $\alpha$, pifithrin- $\alpha$.

\section{Discussion}

Mitochondria not only produce cellular energy through the oxidation of nutrients, but also generate reactive oxygen species (ROS) under pathologic conditions that induce mitochondrial dysfunction [10]. The generation of ROS in conjunction with endoplasmic reticulum (ER) stress induces cellular stress and, consequently, cell death [11,12]. Because the existence of damaged and aged mitochondria threatens cells, the turnover processes of mitochondrial degradation and biogenesis are essential for maintenance of cellular integrity [2-4,13]. With advances in the understanding of autophagy, mitophagy, the selective degradation of mitochondria by autophagy, has garnered attention in mitochondrial metabolism. Conventionally, metformin has been viewed as a type of anti-diabetic drug belonging to the biguanide class that functions by disturbing complex I of the electron transport chain, thereby resulting in activation of $5^{\prime}$ adenosine monophosphate-activated protein kinase (AMP-activated protein kinase) and induction of the activation of NAD+-dependent deacetylase SIRT1 (sirtuin 1) [14,15]. Recently, we demonstrated that metformin activates lipophagy, resulting in relief of hepatosteatosis due to induction of SIRT1-mediated autophagy independent of 
AMP-activated protein kinase [1]. Based on these reports, we hypothesized that metformin plays additional roles in mitochondrial homeostasis through the activation of mitophagy, resulting in an improvement in hepatosteatosis.

Among the cell stress or apoptotic pathways, p53 is known to utilize many mechanisms in its anticancer function and to play roles in apoptosis and genomic stability. Recent studies have reported that cytosolic p53 contributes to mitochondrial integrity and mitophagy [6,7]. Cytosolic p53 has been shown to inhibit the removal of damaged mitochondria through an inhibitory interaction with Parkin, thereby inducing mitochondrial dysfunction [8,9]. Based on these reports, we studied the interplay of metformin in mitophagy and p53 abundance. We therefore investigated (1) whether metformin alleviates the glucolipotoxicity induced cell stress by assessing the expression of ER stress markers and p53 protein; (2) whether Parkin-mediated mitophagy is inhibited by glucolipotoxicity-induced cytosolic p53; and (3) whether metformin induces mitophagy through restoration of the suppressed Parkin-mediated mitophagy induced by increased cytosolic ER stress and p53.

With respect to glucolipotoxicity-induced hepatocellular toxicity and the role of metformin in alleviating glucolipotoxicity, we observed a dose-dependent increase in p53 protein expression in HepG2 cells treated with palmitate in the presence of $35 \mathrm{mM}$ glucose, as well as a dose-dependent decrease in p53 expression in HepG2 cells treated with metformin. Additionally, we found that metformin alleviated glucolipotoxicity-induced p53 overexpression. Similar to these findings, metformin alleviated glucolipotoxicity-induced ER stress, as assessed by Western blotting using ATF6 and Xbp-1 antibodies. To further demonstrate ER stress pathway-mediated glucolipotoxicity, we treated HepG2 cells with the well-established ER stressor thapsigargin (TG). In these experiments, we observed a dose-dependent upregulation of cytosolic p53 and the ER stress markers ATF6 and Xbp-1 in TG-treated HepG2 cells. From a translational point of view [16], nutritional deprivation through caloric restriction (CR) and metformin administration in $o b / o b$ mice also showed significantly decreased levels of cytosolic p53 and the ER stress markers ATF6 and Xbp-1 compared to ad libitum fed $o b / o b$ mice. Endoplasmic reticulum (ER) plays a role as a nutrient sensor in cells, and excess fuel can induce ER stress, which triggers the unfolded protein response (UPR). The UPR leads to both translational attenuation of new protein synthesis and transcriptional activation of stress-response genes to relieve ER stress. However, the UPR also initiates death signals, which function when the stress is pathologically prolonged [17]. ER stress has been generally shown to be inhibited by metformin $[18,19]$, thereby preventing cell injury and apoptosis. Based on results in this study, we suggest that increased expression of p53 accompanies the ER stress pathway.

With respect to mitophagy in hepatic cells, $o b / o b$ mice treated with metformin or CR showed increased mitophagy, as demonstrated by autophagic double membranes surrounding abnormal mitochondria or by the presence of an autophagic vacuole containing mitochondria. In contrast, $o b / o b$ mice under metabolic stress caused by ad libitum feeding of chow diets showed numerous mitochondrial spheroids, as demonstrated by the compressed mitochondrion and lost cristae and matrix. To further validate mitophagy induction by metformin treatment, we additionally demonstrated increased co-localization of the autophagy marker GFP-LC3 and the selective mitochondrial probe MitoTracker Red in metformin-treated HepG2 cells. Mitochondrial spheroids are described as having a unique ring-like morphology with a squeezed mitochondrial matrix. Under various pathophysiologic stresses, mitochondria can undergo direct remodeling to form mitochondrial spheroids, which requires the presence of ROS and either mitofusin1 (Mfn1) or mitofusin2 (Mfn2). In this process, Parkin prevents mitochondrial spheroid formation by causing proteasomal degradation of Mfn1 and Mfn2, which are required for mitophagy [20]. Mitochondrial spheroids can envelop the contents of the cytosol, including damaged mitochondria. Regarding fat accumulation in the liver, each hepatocyte contains about 800 mitochondria, and mitochondrial dysfunction can contribute to the development of NAFLD [21-23]. In a recent study, Ibdah et al., using heterozygous mice deficient in mitochondrial trifunctional protein, found that impairment in $\beta$-oxidation resulted in hepatic steatosis [24]. These alterations were also demonstrated in Otsuka Long-Evans Tokushima 
Fatty (OLETF) rats, which is a model of type 2 diabetes and obesity [25]. Based on the results of our study, we suggest that the metformin-induced autophagic machinery might play important roles in alleviating hepatosteatosis through the induction of both lipophagy and mitophagy.

With respect to the inverse relationship between metformin-induced mitophagy induction and p53 protein abundance [26], the present study showed that glucolipotoxicity inhibited the translocation of Parkin from the cytosol to mitochondria. In contrast, we also showed that metformin or PFT- $\alpha$, which is the p53 inhibitor, induced translocation of Parkin from the cytosol to mitochondria by decreasing the inhibitory interaction with cytosolic p53. These results were additionally validated by examining protein-protein interactions between p53 and Parkin using immunoprecipitates (IP) of endogenous Parkin and p53, as well as the co-localization of autophagy marker GFP-LC3 and the selective mitochondrial probe Mitotracker Red in PFT- $\alpha$-treated HepG2 cells. Regarding the role of mitofusin in mitochondrial spheroid formation, ROS and either Mfn1 or Mfn2 are required. The inner membrane protein optic atrophy 1 (OPA1) might not be important for this process because it is rapidly degraded upon mitochondrial depolarization. Ding et al. reported that Parkin promotes mitophagy through the proteasomal degradation of mitofusins and, consequently, the inhibition of mitochondrial spheroid formation [20]. We confirmed the negative relationship between Parkin and mitofusins in mitochondrial quality control in response to metformin treatment in this study. Based on these results, we suggest that metformin treatment facilitates Parkin-mediated mitophagy instead of mitochondrial spheroid formation by decreasing the inhibitory interaction with cytosolic p53 and increasing the degradation of mitofusins.

The limitation of this study is that we did not conduct in vivo experiments with genetically engineered animal models, such as Parkin knockout mice, to provide a more convincing mechanistic role of metformin in mitophagy. Consequently, these studies should be performed in the future. However, to the best of our knowledge, our study is the first to investigate the molecular interplay among glucolipotoxicity-induced ER stress, cytosolic p53, and Parkin-mediated mitophagy in response to metformin treatment. To summarize our findings, metformin treatment might facilitate Parkin-mediated mitophagy instead of mitochondrial spheroid formation by decreasing the inhibitory interaction with cytosolic p53 and increasing the degradation of mitofusins. This working thesis is summarized in Figure 5.

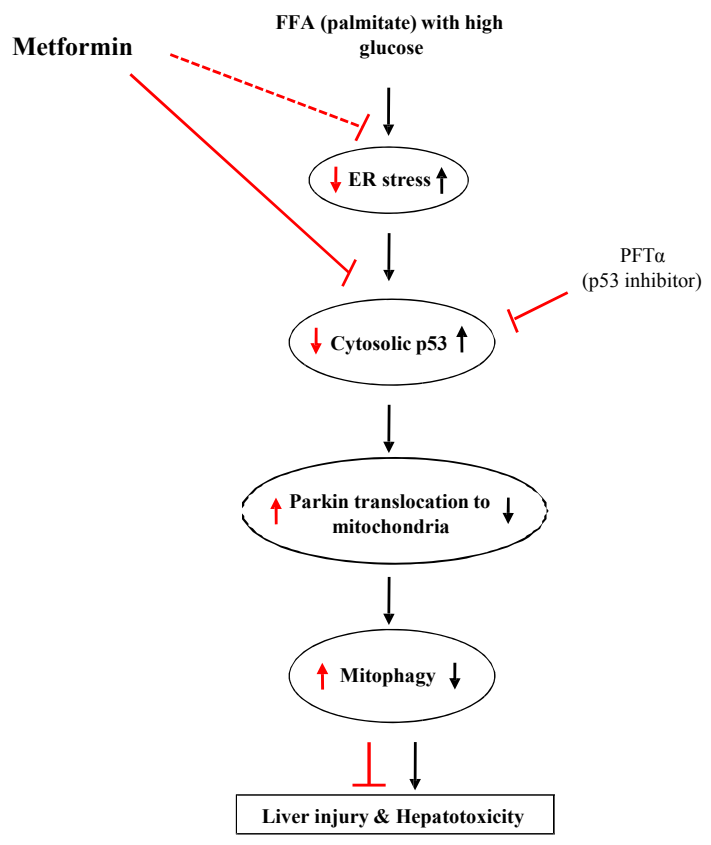

Figure 5. Summary of the working thesis of this study. See the text for details. Black and red arrows indicate the effects of glucolipotoxicity and the drugs (metformin or PFT $\alpha$ ), respectively. 


\section{Experimental Section}

\subsection{Animal and Experimental Procedures}

Eight-week-old $o b / o b$ mice (C57bl/6j background, Jackson Laboratory, Bar Harbor, ME, USA) were maintained at $60 \% \pm 5 \%$ relative humidity and $22 \pm 2{ }^{\circ} \mathrm{C}$ with a 12 -h light/dark cycle over a four-week course of the following treatment regimens: (1) ad libitum feeding of chow diet (Dyets Inc.,

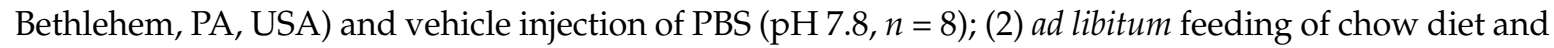
intraperitoneal injections of $300 \mathrm{mg} / \mathrm{kg}$ metformin (Merck KGaA, Darmstadt, Germany, $n=8$ ); and (3) caloric restriction $(\mathrm{CR})$ group $(3 \mathrm{~g} /$ day, $n=8)$. After each animal was sacrificed, fresh liver tissues were fixed with $2 \%$ glutaraldehyde- $2 \%$ paraformaldehyde buffered with $0.1 \mathrm{M}$ phosphate buffer $(\mathrm{pH} 7.2)$ overnight at $4{ }^{\circ} \mathrm{C}$, post-fixed with $1 \%$ osmium tetroxide in a $0.1 \mathrm{M}$ sodium cacodylate buffer ( $\mathrm{pH} 7.4$ ) for $1 \mathrm{~h}$ at room temperature, and dehydrated with a graded series of ethanol, as previously described [5]. The dehydrated tissues were then embedded in Epon, using poly/Bed812 Embedding kit/ DMP-30 (Polysciences, Warrington, PA, USA). All experimental procedures performed in this study followed the ethical guidelines for animal studies and were approved by the Institutional Animal Care and Use Committee of Yonsei University College of Medicine (IACUC No. 2011-0302-1).

\subsection{Cell Culture and Preparation of Subcellular Fractions}

The human hepatoma cell line HepG2 was cultured in Dulbecco's modified Eagle's medium (Welgene, Daegu, Korea) supplemented with 10\% FBS and antibiotics. HepG2 cells were washed and incubated for $10 \mathrm{~min}$ in ice-cold PBS, scraped into PBS with $1 \mathrm{mM}$ phenylmethylsulfonylfluoride (PMSF), and pelleted by centrifugation $(500 \times \mathrm{g})$ at $4{ }^{\circ} \mathrm{C}$ for $10 \mathrm{~min}$. Cell pellets were resuspended in RIPA buffer (Life Science, Berkeley, CA, USA) with protease inhibitors (Thermo Scientific, Waltham, MA, USA), passed 10 times through a 30-gauge syringe to break open the cells, and incubated on ice for $10 \mathrm{~min}$ to generate a whole cell lysate. After centrifugation $(10,000 \times g$, in a microcentrifuge) at $4{ }^{\circ} \mathrm{C}$ for $30 \mathrm{~min}$, the supernatants were collected as whole extracts. Fractionation to separate the mitochondrial and cytosolic fractions was carried out as previously described [27]. Briefly, HepG2 cells were harvested, washed in PBS, and resuspended in cytosolic extraction buffer ( $250 \mathrm{mM}$ sucrose, $70 \mathrm{mM}$ $\mathrm{KCl}, 137 \mathrm{mM} \mathrm{NaCl}, 4.3 \mathrm{mM} \mathrm{Na}_{2} \mathrm{HPO}_{4}, 1.4 \mathrm{mM} \mathrm{KH}_{2} \mathrm{PO}_{4}, \mathrm{pH}$ 7.2, $200 \mu \mathrm{g} / \mathrm{mL}$ digitonin, $100 \mu \mathrm{M}$ PMSF, protease inhibitor cocktail) (Sigma, St. Louis, MO, USA) for $5 \mathrm{~min}$ on ice. Digitonin-permeabilized cells were confirmed by staining with $0.2 \%$ trypan blue solution. Cells were centrifuged at $1000 \times g$ for $5 \mathrm{~min}$; the supernatant was saved as the cytosolic fraction, and the pellet was solubilized in two volumes of mitochondrial lysis buffer ( $50 \mathrm{mM}$ Tris- $\mathrm{HCl}$ pH 7.4, $150 \mathrm{mM} \mathrm{NaCl}, 2 \mathrm{mM}$ EDTA, $2 \mathrm{mM}$ EGTA, $0.2 \%(v / v)$ Triton X-100, 0.3\% NP-40, PMSF, protease inhibitor cocktail) (Sigma) for $5 \mathrm{~min}$ on ice. Cells were then centrifuged at $10,000 \times \mathrm{g}$ for $10 \mathrm{~min}$ at $4{ }^{\circ} \mathrm{C}$, and the supernatant was collected as the mitochondrial extract.

\subsection{Detection of Mitophagy}

For the detection of autophagosomes and mitochondrial spheroids using electron microscopy, sectioning for electron microscopic examination was accomplished with an ultramicrotome (Leica, EM UC7, Wetzlar, Germany), and electron microscopy was performed with a JEM-1011 transmission electron microscope (JEOL). Detection of mitophagy were also performed by transfecting HepG2 cells with a plasmid driving the expression of GFP-LC3 (excitation wavelength $488 \mathrm{~nm}$, emission filter $530 \mathrm{~nm}$ ) (OriGene Technologies, Inc., Rockville, MD, USA) for $16 \mathrm{~h}$, followed by loading of cells with Mitotracker Red (500 nM) (excitation wavelength $488 \mathrm{~nm}$, emission filter $585 \mathrm{~nm}$ ) (Invitrogen, Carlsbad, CA, USA). Transfected cells were fixed with $4 \%$ paraformaldehyde and then observed with a confocal microscope. 


\subsection{Immunofluorescence Staining}

To detect Parkin expression on mitochondria, immunofluorescence staining was performed on cells using Parkin antibody and MitoTracker Red. The cells were stained with MitoTracker Red (500 nM) and fixed with $4 \%$ paraformaldehyde. The fixed cells were incubated with Parkin antibody for $2 \mathrm{~h}$, followed by incubation with secondary antibody (Alexa 488, excitation wavelength $495 \mathrm{~nm}$, emission filter $519 \mathrm{~nm}$ ) (Invitrogen) for $2 \mathrm{~h}$. Next, the cells were observed under the confocal microscope.

\subsection{Free Fatty Acid (FFA) Preparation}

FFA solutions were prepared as previously described [15]. Briefly, $100 \mathrm{mM}$ palmitate (PA) (Sigma) stocks were prepared in $0.1 \mathrm{M} \mathrm{NaOH}$ at $70^{\circ} \mathrm{C}$ and filtered. A one percent (weight/volume) palmitate-free BSA (Sigma) solution was prepared in serum-free DMEM. After the palmitate dissolved, the palmitate solutions were added to serum-free DMEM containing BSA. The $5 \mathrm{mM}$ palmitate $/ 1 \%$ BSA solution was prepared by complexing the appropriate amounts of palmitate to $1 \% \mathrm{BSA}$ in a $55{ }^{\circ} \mathrm{C}$ water bath.

\subsection{Cell Viability Assay}

HepG2 cells were dispensed in wells of 24 -well plates at a density of $5 \times 10^{4}$ cells/well. HepG2 cells were pretreated with $0.5 \mathrm{mM}$ metformin for $3 \mathrm{~h}$ and incubated in the presence or absence of thapsigargin for $24 \mathrm{~h}$. The cells were then treated with $10 \mu \mathrm{L}$ of cell counting kit-8 (CCK-8) solution (Sigma-Aldrich, St. Louis, MO, USA) at $37{ }^{\circ} \mathrm{C}$ for the indicated times, according to the manufacturer's instructions. Absorbance was measured at $450 \mathrm{~nm}$ using a microplate reader (Molecular Devices, Sunnyvale, CA, USA).

\subsection{Western Blotting and Antibodies}

Mouse livers and HepG2 cells were lysed in PRO-PREPTM protein extraction solution (iNtRON Biotechnology, Kyungki-Do, Korea), and the protein contents of the resulting lysates were measured using the Bradford assay (Bio-Rad, Berkeley, CA, USA). Equal amounts of protein were resolved using SDS-PAGE and were electroblotted onto a nitrocellulose membrane (Bio-Rad). Membranes were subsequently probed as indicated with the following primary antibodies: p53 (Cell Signaling, Danvers, MA, USA), ATF6, sXBP1, GAPDH, Parkin, mitofusin (Mfn1) (Santa Cruz Biotechnology, Santa Cruz, CA, USA), and COXVI (Life Science). Secondary antibodies (anti-rabbit and anti-mouse) were from Santa Cruz Biotechnology.

\subsection{Co-Immunoprecipitation Assay}

For co-immunoprecipitation (IP) assays, HepG2 cells and liver tissue were harvested in lysis buffer (50 mM Tris (pH 7.4), $140 \mathrm{mM} \mathrm{NaCl}, 1 \%$ Triton X-100, $30 \mathrm{M} \mathrm{MG132,} \mathrm{and} \mathrm{protease} \mathrm{inhibitors).} \mathrm{Cell}$ extracts were centrifuged, and the supernatants were incubated with anti-p53 or anti-Parkin antibodies for $12 \mathrm{~h}$ at $4{ }^{\circ} \mathrm{C}$. ProteinA/G PLUS-Agarose (Santa Cruz Biotechnology) was then added to each sample, and incubation was carried out overnight at $4{ }^{\circ} \mathrm{C}$ on a rotating device. Immunoprecipitates were collected by centrifugation at $1000 \times \mathrm{g}$ for $5 \mathrm{~min}$ at $4^{\circ} \mathrm{C}$ and washed with lysis buffer containing $500 \mathrm{mM} \mathrm{NaCl}$ or PBS. The pellets were eluted by heating at $95^{\circ} \mathrm{C}$ for $5 \mathrm{~min}$ in $1 \times$ electrophoresis sample buffer.

\subsection{Statistical Analysis}

Statistical analysis was performed using PRISM (GraphPad Software Inc., San Diego, CA, USA). Results are expressed as mean $\pm \mathrm{SE}$, and statistical significance was calculated using Student's $t$-test. Alternatively, for comparisons involving more than two groups, one-way analysis of variance (ANOVA) with a post hoc Bonferroni multiple comparison test was used to assess the differences. Statistical significance was defined as the conventional $p$-value of $<0.05$. 


\section{Conclusions}

In conclusion, the present study suggests that metabolic stresses in $o b / o b$ mice induce mitochondrial spheroid formation and ultimately result in fat droplet accumulation in hepatocytes. In contrast, metformin treatment of $o b / o b$ mice induces Parkin-mediated mitophagy upregulation by inhibiting glucolipotoxicity-induced cytosolic p53.

Acknowledgments: This research was supported by the Basic Science Research Program through the National Research Foundation of Korea (NRF) funded by the Ministry of Education, Science and Technology (2014R1A1A2057906).

Author Contributions: Young Mi Song and Woo Kyung Lee performed experiments, analyzed the data, and wrote and edited the manuscript; Byung-Wan Lee was responsible for experimental design and initiated, planned and supervised the study; Yong-ho Lee, Eun Seok Kang and Bong-Soo Cha have given much advice and technological support. All authors approved the final version of the article, including the authorship list.

Conflicts of Interest: The authors declare no conflict of interest.

\section{References}

1. Song, Y.M.; Lee, Y.H.; Kim, J.W.; Ham, D.S.; Kang, E.S.; Cha, B.S.; Lee, H.C.; Lee, B.W. Metformin alleviates hepatosteatosis by restoring sirt1-mediated autophagy induction via an amp-activated protein kinase-independent pathway. Autophagy 2015, 11, 46-59. [CrossRef] [PubMed]

2. Franz, A.; Kevei, E.; Hoppe, T. Double-edged alliance: Mitochondrial surveillance by the ups and autophagy. Curr. Opin. Cell Biol. 2015, 37, 18-27. [CrossRef] [PubMed]

3. Zhu, J.; Wang, K.Z.; Chu, C.T. After the banquet: Mitochondrial biogenesis, mitophagy, and cell survival. Autophagy 2013, 9, 1663-1676. [CrossRef] [PubMed]

4. Tanida, I. Autophagosome formation and molecular mechanism of autophagy. Antioxid. Redox Signal. 2011, 14, 2201-2214. [CrossRef] [PubMed]

5. Ren, J.; Pulakat, L.; Whaley-Connell, A.; Sowers, J.R. Mitochondrial biogenesis in the metabolic syndrome and cardiovascular disease. J. Mol. Med. 2010, 88, 993-1001. [CrossRef] [PubMed]

6. Suzuki, S.; Tanaka, T.; Poyurovsky, M.V.; Nagano, H.; Mayama, T.; Ohkubo, S.; Lokshin, M.; Hosokawa, H.; Nakayama, T.; Suzuki, Y.; et al. Phosphate-activated glutaminase (gls2), a p53-inducible regulator of glutamine metabolism and reactive oxygen species. Proc. Natl. Acad. Sci. USA 2010, 107, 7461-7466. [CrossRef] [PubMed]

7. Vousden, K.H.; Ryan, K.M. P53 and metabolism. Nat. Rev. Cancer 2009, 9, 691-700. [CrossRef] [PubMed]

8. Hoshino, A.; Mita, Y.; Okawa, Y.; Ariyoshi, M.; Iwai-Kanai, E.; Ueyama, T.; Ikeda, K.; Ogata, T.; Matoba, S. Cytosolic p53 inhibits Parkin-mediated mitophagy and promotes mitochondrial dysfunction in the mouse heart. Nat. Commun. 2013, 4, 2308. [CrossRef] [PubMed]

9. Hoshino, A.; Ariyoshi, M.; Okawa, Y.; Kaimoto, S.; Uchihashi, M.; Fukai, K.; Iwai-Kanai, E.; Ikeda, K.; Ueyama, T.; Ogata, T.; et al. Inhibition of p53 preserves Parkin-mediated mitophagy and pancreatic $\beta$-cell function in diabetes. Proc. Natl. Acad. Sci. USA 2014, 111, 3116-3121. [CrossRef] [PubMed]

10. Ross, J.M.; Olson, L.; Coppotelli, G. Mitochondrial and ubiquitin proteasome system dysfunction in ageing and disease: Two sides of the same coin? Int. J. Mol. Sci. 2015, 16, 19458-19476. [CrossRef] [PubMed]

11. Chae, M.K.; Park, S.G.; Song, S.O.; Kang, E.S.; Cha, B.S.; Lee, H.C.; Lee, B.W. Pentoxifylline attenuates methionine- and choline-deficient-diet-induced steatohepatitis by suppressing TNF- $\alpha$ expression and endoplasmic reticulum stress. Exp. Diabetes Res. 2012, 2012. [CrossRef] [PubMed]

12. Song, Y.M.; Song, S.O.; You, Y.H.; Yoon, K.H.; Kang, E.S.; Cha, B.S.; Lee, H.C.; Kim, J.W.; Lee, B.W. Glycated albumin causes pancreatic $\beta$-cells dysfunction through autophagy dysfunction. Endocrinology 2013, 154, 2626-2639. [CrossRef] [PubMed]

13. Pyo, J.O.; Yoo, S.M.; Jung, Y.K. The interplay between autophagy and aging. Diabetes Metab. J. 2013, 37, 333-339. [CrossRef] [PubMed]

14. Caton, P.W.; Nayuni, N.K.; Kieswich, J.; Khan, N.Q.; Yaqoob, M.M.; Corder, R. Metformin suppresses hepatic gluconeogenesis through induction of SIRT1 and GCN5. J. Endocrinol. 2010, 205, 97-106. [CrossRef] [PubMed] 
15. Song, Y.M.; Song, S.O.; Jung, Y.K.; Kang, E.S.; Cha, B.S.; Lee, H.C.; Lee, B.W. Dimethyl sulfoxide reduces hepatocellular lipid accumulation through autophagy induction. Autophagy 2012, 8, 1085-1097. [CrossRef] [PubMed]

16. Wang, Y. Molecular links between caloric restriction and Sir2/SIRT1 activation. Diabetes Metab. J. 2014, 38, 321-329. [CrossRef] [PubMed]

17. Delaunay-Moisan, A.; Appenzeller-Herzog, C. The antioxidant machinery of the endoplasmic reticulum: Protection and signaling. Free Radic. Biol. Med. 2015, 83, 341-351. [CrossRef] [PubMed]

18. Simon-Szabo, L.; Kokas, M.; Mandl, J.; Keri, G.; Csala, M. Metformin attenuates palmitate-induced endoplasmic reticulum stress, serine phosphorylation of IRS-1 and apoptosis in rat insulinoma cells. PLoS ONE 2014, 9, e97868. [CrossRef] [PubMed]

19. Jung, T.W.; Lee, M.W.; Lee, Y.J.; Kim, S.M. Metformin prevents endoplasmic reticulum stress-induced apoptosis through AMPK-PI3K-c-Jun $\mathrm{NH}_{2}$ pathway. Biochem. Biophys. Res. Commun. 2012, 417, 147-152. [CrossRef]

20. Ding, W.X.; Guo, F.; Ni, H.M.; Bockus, A.; Manley, S.; Stolz, D.B.; Eskelinen, E.L.; Jaeschke, H.; Yin, X.M. Parkin and mitofusins reciprocally regulate mitophagy and mitochondrial spheroid formation. J. Biol. Chem. 2012, 287, 42379-42388. [CrossRef] [PubMed]

21. Yoo, H.J.; Choi, K.M. Hepatokines as a link between obesity and cardiovascular diseases. Diabetes Metab. J. 2015, 39, 10-15. [CrossRef] [PubMed]

22. Sanyal, A.J.; Campbell-Sargent, C.; Mirshahi, F.; Rizzo, W.B.; Contos, M.J.; Sterling, R.K.; Luketic, V.A.; Shiffman, M.L.; Clore, J.N. Nonalcoholic steatohepatitis: Association of insulin resistance and mitochondrial abnormalities. Gastroenterology 2001, 120, 1183-1192. [CrossRef] [PubMed]

23. Zhou, M.; Xu, A.; Tam, P.K.; Lam, K.S.; Chan, L.; Hoo, R.L.; Liu, J.; Chow, K.H.; Wang, Y. Mitochondrial dysfunction contributes to the increased vulnerabilities of adiponectin knockout mice to liver injury. Hepatology 2008, 48, 1087-1096. [CrossRef] [PubMed]

24. Ibdah, J.A.; Perlegas, P.; Zhao, Y.; Angdisen, J.; Borgerink, H.; Shadoan, M.K.; Wagner, J.D.; Matern, D.; Rinaldo, P.; Cline, J.M. Mice heterozygous for a defect in mitochondrial trifunctional protein develop hepatic steatosis and insulin resistance. Gastroenterology 2005, 128, 1381-1390. [CrossRef] [PubMed]

25. Rector, R.S.; Thyfault, J.P.; Uptergrove, G.M.; Morris, E.M.; Naples, S.P.; Borengasser, S.J.; Mikus, C.R.; Laye, M.J.; Laughlin, M.H.; Booth, F.W.; et al. Mitochondrial dysfunction precedes insulin resistance and hepatic steatosis and contributes to the natural history of non-alcoholic fatty liver disease in an obese rodent model. J. Hepatol. 2010, 52, 727-736. [CrossRef] [PubMed]

26. Nelson, L.E.; Valentine, R.J.; Cacicedo, J.M.; Gauthier, M.S.; Ido, Y.; Ruderman, N.B. A novel inverse relationship between metformin-triggered AMPK-SIRT1 signaling and p53 protein abundance in high glucose-exposed HepG2 cells. Am. J. Physiol. Cell Physiol. 2012, 303, C4-C13. [CrossRef] [PubMed]

27. Adrain, C.; Creagh, E.M.; Martin, S.J. Apoptosis-associated release of Smac/DIABLO from mitochondria requires active caspases and is blocked by Bcl-2. Embo J. 2001, 20, 6627-6636. [CrossRef] [PubMed] 